\title{
Actualización de las recomendaciones sobre el uso de la monitorización ambulatoria de presión arterial. Documento de consenso de la Sociedad Chilena de Hipertensión
}

\author{
Hernán Prat $\mathbf{M}^{1}$, G loria Valdés S, Ó scar Román A, \\ L Hernán Zárate $M$.
}

\section{Update of consensus reccomendations of the Chilean Hypertension Society about ambulatory blood pressure monitoring}

Ambulatory blood pressure monitoring (ABPM) is a valuable tool to evaluate the blood pressure pattern, to identify hypertensive patients, to diagnose white coat and masked hypertension and in situations in which a tight control of hypertension is crucial. This is an update of 1999 consensus recommendations about the use to ABPM, considering that there is new evidence concerning its benefits, and the clinical experience with its application has increased. Equipment programming, its installation, the interpretation and analysis of the data are described, and a report sheet for patients is included. New recommendations have been added to the accepted indications. Normal blood pressure ranges for children and pregnant women have been replaced by new data (Rev Méd Chile 2009; 137: 1235-47).

(Key words: Blood pressure; Consensus; Hypertension)

Recibido el 11 de noviembre, 2008. Aceptado el 19 de junio, 2009.

${ }^{1}$ Coordinador del grupo

E n 1999, nuestra Sociedad elaboró recomendaciones para el uso de la monitorización ambulatoria de presión arterial de 24 h (MAPA) ${ }^{19}$. El tiempo, la mayor experiencia ganada con su uso y los nuevos conceptos referentes a la necesidad de

$\overline{\text { Correspondencia a: Dr. Hernán Prat M. Sociedad Chilena }}$ de Hipertensión. Bernarda Morín 488, $3^{\text {er }}$ piso, Providencia. Casilla 168, correo 55. Santiago 9, Chile. E mail: hipertension@smschile.cl ajustar mejor las presiones en condiciones especiales, han hecho necesaria una puesta al día de ellas, esta vez agregando grados de recomendación y niveles de evidencia (Tabla 1). Sólo pretenden guiar y dar a conocer los conceptos más importantes en el uso de la MAPA, respetando la visión individual de quienes están familiarizados con ella.

La MAPA es una valiosa técnica de apoyo al manejo clínico de la enfermedad hipertensiva, permite un mejor conocimiento de las cifras tensionales en hipertensos, en aquellos con sospe- 
Tabla 1. Grados de recomendación y niveles de evidencia para estas guías ${ }^{16}$

\section{Grados de recomendación}

Cuando sean aplicables, las siguientes clases debieran usarse:

I Cuando hay consenso en la indicación.

IIa Cuando hay un desacuerdo respecto a la indicación, pero se aprueba por la mayoría.

IIb Cuando hay un desacuerdo respecto a la indicación, con opinión dividida.

III Cuando hay consenso en la contraindicación o cuando no es aplicable.

Niveles de Evidencia

A: Estudios clínicos grandes, randomizados y metaanálisis.

B: Estudios clínicos bien diseñados y estudios observacionales.

C: Reporte de casos y series.

D: Publicaciones basadas en consensos y opiniones de expertos.

cha de hipertensión arterial o con coexistencia de otros factores de riesgo cardiovascular. También contribuye a investigar las características que influyen en el pronóstico de la enfermedad hipertensiva (descenso nocturno y eficacia de fármacos). La variabilidad de la presión arterial (PA) en las $24 \mathrm{~h}$ o variación circadiana, es considerable (estimada en aproximadamente $50 \mathrm{mmHg}$ ) y se asocia mejor al pronóstico de daño a los órganos blanco y morbimortalidad cardiovascular que la toma aislada de PA. Esta variación observada en personas normotensas aumenta significativamente en hipertensos, especialmente en adultos mayores y diabéticos ${ }^{1}$.

Las mediciones aisladas de la PA y en horas diferentes para cada paciente, pueden estar sujetas a errores de sub o sobreestimación de acuerdo a factores, entre los cuales se encuentran:

a) La variabilidad de la PA, no sólo en el día, sino que también en lapsos breves de tiempo, lo que es aun más significativo.

b) La medición aislada en la clínica frecuentemente provoca reacciones de alarma del paciente y la PA obtenida puede elevarse, fenómeno conocido como de "bata blanca". Ocurre en aproximadamente $20 \%$ a $30 \%$ de las personas y los estudios sugieren que representa un riesgo cardiovascular intermedio entre normotensos e hipertensos (grado de recomendación IIb, evidencia nivel B). También puede darse lo opuesto, la hipertensión enmascarada, la que ocurre alrededor de $10 \%$ a
$17 \%$ de los hipertensos, si en la consulta médica las presiones son normales, pero son altas fuera de ella2,3.

c) Numerosos estudios han señalado que la presión media en las $24 \mathrm{~h}$ se correlaciona mejor con el daño a los órganos blanco (evidencia nivel A) ${ }^{4}$.

d) Los registros de la PA en el monitoreo ambulatorio son más reproducibles que los casuales o clínicos (grado de recomendación I, evidencia nivel C) ${ }^{5}$.

e) La evidencia epidemiológica señala que la morbimortalidad coronaria y cerebrovascular es mayor al despertar (o período matinal) y se correlaciona con la mayor alza de la PA durante las primeras horas del día 6 .

f) El descenso nocturno (o reposo) de la PA (dip) está ausente entre 17\% y 35\% de los casos en los hipertensos, con un peor pronóstico. A la vez, obliga a descartar hipertensiones secundarias o malignas ${ }^{7}$.

Las ventajas y desventajas del uso de la MAPA se muestran en la Tabla 2. La seguridad de los valores registrados se ha confirmado por protocolos de validación de equipos, efectuados por instituciones internacionales como la AAMI (Asociación para el Avance de Instrumentación Médica) o la BHS (Sociedad Británica de Hipertensión), respaldando así la investigación clínica y control de los pacientes.

Las indicaciones conocidas y consensuadas para solicitar el examen se muestran en Tabla $3 \mathrm{a}$. 
Tabla 2. Ventajas y desventajas de la MAPA en comparación con las tomas aisladas de presión arterial

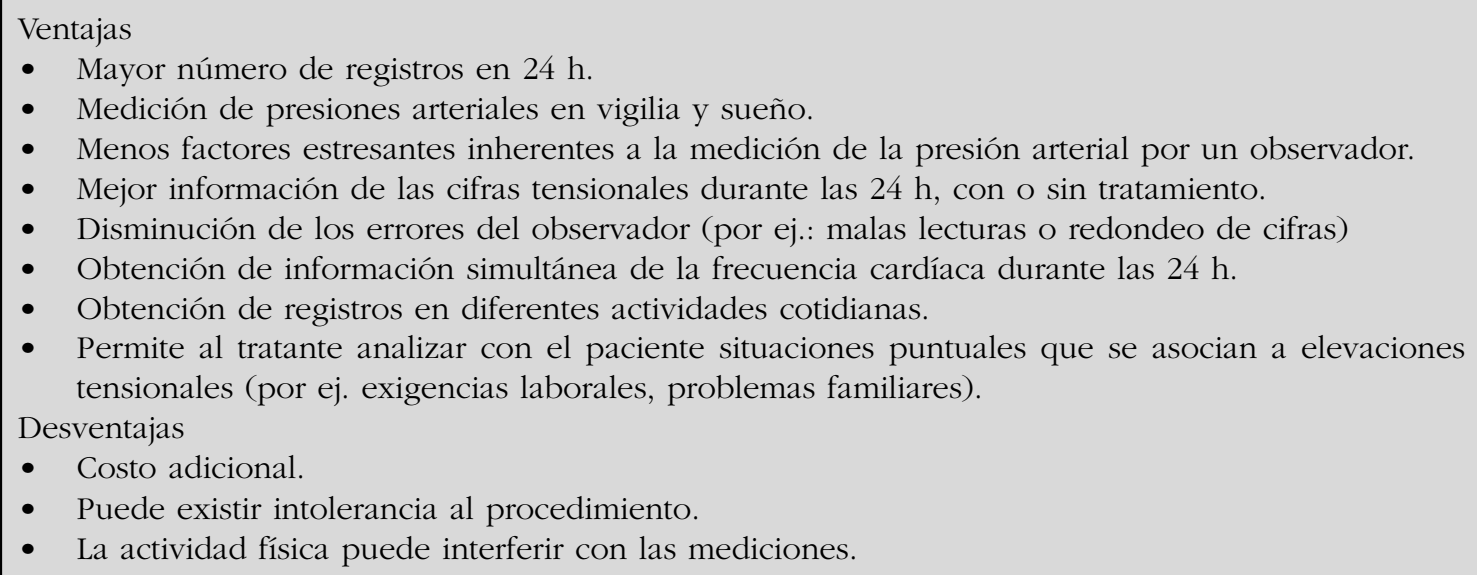

Tabla 3a. Indicaciones de la MAPA

\section{Indicaciones aceptadas}

- Sospecha de hipertensión del "delantal o bata blanca", o "efecto de bata blanca" (grado de recomendación I, evidencia nivel B).

- Hipertensión episódica.

- "Resistencia" a medicamentos antihipertensivos (grado de recomendación IIa, evidencia nivel B).

- Evaluación de síntomas de hipotensión arterial en pacientes con medicación antihipertensiva (grado de recomendación I, evidencia nivel D).

- Evaluación de la medicación en pacientes hipertensos de alto riesgo cardiovascular, (grado de recomendación IIa, evidencia nivel B).

- Evaluación de PA nocturna y presencia de dip.

- Disfunción autonómica

Hemos agregado otras indicaciones que estimamos importantes (Tabla 3b).

Programación de los equipos. El número diario de registros de PA debiera exceder los $50^{8}$. La razón es que, considerando que las conclusiones se toman de promedios, éstos son más fieles si el número de mediciones es mayor. Para obtener este número, los equipos deben programarse: período diurno: $(7-23 \mathrm{~h})$ registros cada 15 ó 20 min; período nocturno: (23-7 h) registros cada 30 ó $60 \mathrm{~min}$. Debieran registrarse al menos 14 tomas válidas en el día y 7 en la noche, mínimo bajo el cual debe repetirse el examen (grado de recomendación I, evidencia nivel B).

Con el fin de precisar mejor el período de dip se ha propuesto evitar horarios de "transición". La Sociedad Europea de Hipertensión sugiere medir el período diurno de 09-21 h y el nocturno de 01-06 h? Esto no está exento de sesgo y debe analizarse esos períodos con el diario que aporta el paciente (debe reportar las horas de sueño, vigilia y la calidad del sueño).

Instalación del monitor ambulatorio. Para entregar una información fidedigna y familiarizar al 
Tabla 3b. Indicaciones adicionales que no tienen consenso pero muy útiles especialmente en hipertensos de alto riesgo

a) Diabéticos con compromiso cardiovascular. En especial el tipo 1.

b) Con enfermedad clínica cardiovascular.

c) Con 2 o más factores mayores de riesgo cardiovascular (además de diabetes).

d) Con compromiso de órgano(s) diana (grado de recomendación I, nivel de evidencia B) :

- Enfermedad arterial periférica (carótidas, extremidades inferiores, etc.).

- Nefropatía crónica.

- Insuficiencia cardiaca.

- Hipertrofia ventricular izquierda.

- Angina, infarto del miocardio o revascularización miocárdica previa.

- Accidente vascular cerebral o isquemia cerebral transitoria.

e) Pacientes en diálisis crónica con hipertensión arterial de difícil manejo, en los cuales se recomienda monitorizar durante todo el periodo interdiálisis.

f) Embarazadas con hipertensión arterial crónica o preeclampsia (grado de recomendación IIa, nivel de evidencia B).

g) Persistencia de síntomas y daño por hipertensión arterial a pesar de un buen tratamiento aparente (obs. hipertensión enmascarada) (grado de recomendación IIb, evidencia nivel D).

h) Sujetos con apnea de sueño comprobada, incluso si son normotensos en la consulta.

i) En pacientes en que se sospecha un feocromocitoma por crisis esporádicas, mientras se recolectan catecolaminas o metanefrinas urinarias. Una determinación negativa durante un día en que se han expresado las alzas de presión y frecuencia cardíaca obliga a buscar otra causa de hipertensión.

j) Evaluación de mayores de 65 años, con lo que se optimiza la estratificación del riesgo y su tratamiento, ya que la rigidez arterial favorece la mayor variabilidad y frecuentemente el fenómeno de "bata blanca" (grado de recomendación IIa, nivel de evidencia A).

paciente con el equipo, es necesario que este sea instalado por médico, enfermera u otro profesional entrenado, siendo fundamental que se le explique el objetivo del examen y el modo en que funcionará el aparato. Se ha constatado que los pacientes motivados y mejor preparados lo toleran mejor, y las presiones serán más representativas de sus valores habituales.

Antes de instalar el equipo es necesario:

1. Tomar la presión en ambos brazos, colocar el monitor en el brazo con mayor presión (grado de recomendación I). Si la diferencia entre ambos es menor a $10 \mathrm{mmHg}$, preferir el brazo no dominante. Si se comprueba la existencia de una diferencia significativa entre ellos, esta acarrea mayor riesgo cardiovascular y debe precisarse este dato en el informe (evidencia nivel B) ${ }^{10}$.

2. Detectar la presencia de extrasístoles frecuentes o de fibrilación auricular, pues producen una variación de las cifras tensionales según sea el período de llenado diastólico.

3. Seleccione el brazalete apropiado según la circunferencia del brazo (grado de recomendación I, evidencia nivel B).

Es importante hacer funcionar el equipo para que el paciente se familiarice a la molestia de la insuflación.

Se debe proporcionar un número telefónico para consultar por molestias o dudas durante el horario de trabajo. Adjuntamos un modelo de recomendaciones impresas (Anexo 1).

ELEMENTOS A CONSIDERAR EN EL INFORME DE UNA MAPA E INTERPRETACIÓN DEL EXAMEN

Información básica:

1. Datos personales: nombre, edad, médico solicitante. 


\section{Anexo 1. Diario de registro de eventos}

Debe anotarse:

1. Hora exacta del evento (coordinada con la del monitor).

2. Tipo de molestia, duración y actividad relacionada.

3. Consumo de alcohol y cigarrillos.

4. Situaciones de estrés.

5. Horario en que toma medicamentos.

6. Horario de acostada y levantada.

7. Horario de traslado hacia y desde el trabajo.

8. Actividades del día ifueron las habituales?

9. Tolerancia al examen y cómo durmió con el monitor instalado.

Ejemplo de diario

INSTRUCCIONES MONITOREO DE PRESIÓN ARTERIAL DE 24 HORAS (“HOLTER")

Este examen se le ha indicado para conocer presiones arteriales mientras realiza sus actividades diarias. Para un diagnóstico efectivo debemos saber lo que usted hace, en el horario en se toma los medicamentos antihipertensivos y las molestias que presente durante el período del estudio.

Por favor anote cada vez que realice una actividad o tenga molestias.

Actividades que interesa:

Caminar — Ejercicios — Fumar — Ingesta de medicamentos

Estados emocionales, (como rabia, sustos, angustia).

Síntomas:

Dolor de cabeza, zumbido de oídos.

Visión de candelillas, "moscas" o puntos.

Ahogos, palpitaciones, mareos, decaimiento.

Ejemplo:

\begin{tabular}{|l|l|l|}
\hline Hora & Actividades & Síntomas \\
\hline $7: 15$ & Me levanté & \\
$8: 30$ & Tomé medicamentos & \\
$9: 40$ & Fumé un cigarrillo & \\
$10: 30$ & Discusión en el trabajo & Dolor de cabeza \\
$12: 30$ & Clase de gimnasia & Zumbidos de ó́dos \\
$16: 30$ & Reunión trabajo & \\
$22: 30$ & Tomé medicamentos, me acosté & \\
\hline $\begin{array}{l}\text { Importante: } \\
\text { - No abra la grabadora. }\end{array}$ & \\
- No desconecte ningún cable. & \\
- No moje la grabadora, sus conexiones o el brazalete. & \\
- Puede afeitarse con máquina eléctrica. & \\
Para lograr el mayor rendimiento de este control continuo de presión arterial le agradeceremos que llene \\
cuidadosamente esta hoja.
\end{tabular}


(continuación Anexo 1)

Nombre completo:

Edad:

Altura:

Peso:

Teléfono:

Dirección:

Médico que solicitó el examen:

Razón para solicitar examen:

Medicamentos (dosis y hora):

$-$

$-$

Llene el siguiente diario de actividades:

\begin{tabular}{l|l|l}
\hline Hora & Actividades & Síntomas \\
\hline & & \\
\hline & & \\
\hline & & \\
\hline & & \\
\hline & & \\
\hline & & \\
\hline
\end{tabular}

(subraye)

Durante el examen me sentí / tranquilo / tenso

Mi tolerancia al examen fue / buena / regular / mala

Tuve mayor / igual / menor / actividad que un día normal

Tuve mayor / igual / menor / preocupaciones que un día normal

Dormí / tranquilo / interrumpido / no pude dormir / debido al examen / patrón habitual de sueño.

RECUERDE: Usted es portador de un equipo delicado de diagnóstico. Cuídelo. Cada vez que sienta el aviso de toma de presión, deje de hacer actividades con el brazo. Deberá concurrir a retirarse el equipo al Laboratorio de....

A las:

del día:

- Si es posible, señalar la altura en menores de 18 años.

2. Medicación recibida: especificar fármacos, horarios, dosis.

3. Programación del monitor: períodos diurno, nocturno (registros cada cuantos minutos).

4. Resultados:

- Número de registros totales, número de registros válidos.

- Promedios de presiones en las $24 \mathrm{~h}$, diurnas y nocturnas.
- Horarios en que se agrupan las mayores presiones; optativamente pueden calcularse promedios para períodos específicos.

- Presiones máximas y mínimas en esos períodos.

- Porcentaje de cargas diurnas y nocturnas.

- Presencia de descenso nocturno normal de la presión (dip).

- Presión de pulso.

- Frecuencia cardiaca, promedios y rangos. 


\section{INTERPRETACIÓN DEL EXAMEN}

Validación del estudio: Se recomienda repetir el examen si hay:

- Pocos registros (menos de 40).

- Pérdida de registros por más de 2 h. Puede aceptarse hasta $3 \mathrm{~h}$ si se observa uniformidad de valores de presiones previas y posteriores.

- Condiciones inhabituales del paciente durante el examen, que determinen cambios de presión arterial (ej. fiebre, enfermedades intercurrentes, estrés, ejercicios no habituales).

- 20\% o más registros considerados erróneos.

\section{Análisis de resultados:}

a) Rechazo de registros

Los monitores están programados para rechazar registros que se consideren erróneos y repetirlos. Debemos considerar erróneos aquellos registros que:

- Sean aislados, anormalmente altos o bajos (PAS $>250$ o $<70 \mathrm{mmHg}$ o PAD $>150$ o $<40$ $\mathrm{mmHg}$ ).

- Tengan valores excesivos al inicio o término del examen (y el resto de ellos sean uniformes).

- Su presión de pulso sea $>150$ mmHg o $<20$ mmHg.

- Se asocie a frecuencias cardíacas $>160$ o $<35$ por min.

Adicionalmente, recomendamos descartar registros aislados que varíen en más de 30\% de los valores previos o posteriores.

El rechazo de registros puede modificar los resultados de promedios, principalmente los sistólicos, y la variabilidad de las cifras tensionales. Puede reducir los promedios sistólicos en 4 $\mathrm{mmHg}$ respecto a los no corregidos. Un estudio ${ }^{11}$ mostró que la prevalencia de hipertensión sostenida disminuyó entre 6\% y 10\% cuando se usaban estos métodos de "corrección". b) Análisis de la presión arterial

De acuerdo a lo sugerido por la American Heart Association (AHA) ${ }^{12}$, los valores promedios de las cifras entregadas por el monitor se clasifican en la Tabla 4 (grado de recomendación I).

La clasificación de la AHA deja algunos hiatos entre lo "normal" y "anormal", porque no ha habido un acuerdo general si los valores intermedios definen una hipertensión. Debe considerarse al paciente en forma integral, analizando: edad (no es igual un menor de 40 años con gran posibilidad de evolucionar a una hipertensión establecida, que uno de 75 años sin antecedentes), factores de riesgo asociados, si está con hipotensores y condiciones del examen. Si persiste la duda, recomendamos repetir el monitoreo.

Agregamos la clasificación de normo e hipertensión del sistema DABL (Disturbance Amplification in Boundary Layers), una plataforma de manejo de datos y registros asistida con expertos internacionales en MAPA (Anexo 2 b). También tiene un registro de la validación de equipos para MAPA, (página web http:// www.dableducational.com).

c) Presión de pulso (diferencia entre presión sistólica-diastólica).

Promedio en $24 \mathrm{~h}$ :

Normal: hasta $50 \mathrm{mmHg}$.

Anormal: sobre $55 \mathrm{mmHg}$.

La presión de pulso anormal aumenta el riesgo cardiovascular $^{13}$. Es frecuente encontrarla en ancianos por rigidez arterial y es difícil de tratar. Al menos la terapéutica no debe aumentarla.

d) Cargas.

Aunque la carga hipertensiva debiera representar el área de presiones que sobrepasan el límite superior de tolerancia, según autores que la denominan "índice hiperbárico" ${ }^{14}$, en la

Tabla 4. Clasificación de las presiones según la AHA: (en mmHg)

\begin{tabular}{|lrrr|}
\hline & Óptimo & Normal & Anormal \\
\hline Promedio $24 \mathrm{~h}$ & $<125 / 75$ & $<130 / 80$ & $>135 / 85$ \\
Diurno & $<130 / 80$ & $<135 / 85$ & $>140 / 90$ \\
Nocturno & $<115 / 65$ & $<120 / 70$ & $>125 / 75$ \\
\hline
\end{tabular}


Anexo 2a. Clasificación de etapas de hipertensión de acuerdo a clasificación del JNC 7

\begin{tabular}{|lc|}
\hline Hipertensión arterial Etapa 1: & Hipertensión arterial Etapa 2: \\
\hline $\begin{array}{l}\text { Sistólica: } \geq 130-154 \mathrm{mmHg} \\
\text { Diastólica: } \geq 80-94 \mathrm{mmHg}\end{array}$ & $\geq 155 \mathrm{mmHg}$ \\
$\geq 95 \mathrm{mmHg}$
\end{tabular}

Anexo 2b. Reporte Dabl

\begin{tabular}{|c|c|c|c|c|c|c|}
\hline \multicolumn{7}{|c|}{ Rangos para definiciones usadas en los reportes Dabl ${ }^{\circledR}$ para monitoreo ambulatorio de presión arterial } \\
\hline & \multicolumn{6}{|c|}{ Hipertensión (mmHg) } \\
\hline & Baja & Normal & Limítrofe & Leve & Moderada & Severa \\
\hline \multicolumn{7}{|l|}{ Diurnas } \\
\hline Presión sistólica & $<100$ & $100-135$ & $136-140$ & $141-155$ & $156-170$ & $>170$ \\
\hline Presión diastólica & $<65$ & $65-85$ & $86-90$ & $91-100$ & $101-110$ & $>110$ \\
\hline \multicolumn{7}{|l|}{ Nocturnas } \\
\hline Presión sistólica & $<90$ & $91-120$ & $121-125$ & $126-135$ & $136-150$ & $>150$ \\
\hline Presión diastólica & $<50$ & $51-70$ & $71-75$ & $76-85$ & 86-100 & $>100$ \\
\hline
\end{tabular}

www.dabl.ie/en/prod_abpm.aspx

práctica se considera el porcentaje de elevaciones por sobre las cifras normales fijadas para el período diurno y nocturno. Encontramos adecuada esta clasificación:

- Anormal: $>40 \%$ de registros. Sobre esta cifra hay alta correlación con el daño en órganos diana ${ }^{15}$.

- Normal: 0\%-15\%

- No significativa: $16 \%-25 \%$

- Limítrofe: 26\%-39\%

Para el cómputo de las cargas, se debe programar los equipos con 135/85 mmHg en horas diurnas y con 120/80 $\mathrm{mmHg}$ en horas nocturnas. Las cargas apoyan los criterios de severidad del examen, pero en ningún caso hacen diagnóstico por sí solas.

e) Ciclo circadiano

Analizar los cambios de presión que ocurren en las $24 \mathrm{~h}$ y la relación de ellos con la medicación dada al paciente.

El dip nocturno (de reposo o descenso nocturno de la PA) debe considerarse positivo o presente si la caída de la presión media (PAM) es mayor del 10\% de aquélla del período diurno.
- Normal: con caída de la PAM entre 10\% y $20 \%$.

- Exagerado: con caídas de la PAM mayores a $20 \%$.

- Invertido (o Riser): si la PAM nocturna es mayor que la PAM diurna.

- Algunos consensos han introducido el concepto de dip "atenuado" cuando hay caídas de la PAM nocturna entre 0,1\% y 9,9\% argumentando que es un continuo y que las cifras arbitrarias no corresponden a procesos biológicos ${ }^{16}$. A nuestro parecer, podrían incluirse en esta calificación a las caídas de PAM entre 5\% y 9,9\%.

Debe expresarse tanto el dip sistólico como el diastólico si se toman estas cifras en vez de la PAM, privilegiando el sistólico si no hay correspondencia entre ambos valores.

Siempre es necesario analizar la curva de las presiones en $24 \mathrm{~h}$, ya que el dip puede estar presente, pero acortado o desplazado (averiguar la hora en que el sujeto se acuesta, o si durmió adecuadamente) y erróneamente podemos considerarlo ausente si sólo analizamos los promedios. 
La ausencia de dip se ha correlacionado con mayor morbimortalidad cardiovascular ${ }^{17}$. Aunque es beneficioso presentar dip, la presencia de uno exagerado podría asociarse a complicaciones de cardiopatía coronaria u otras que impliquen hipoperfusión nocturna.

El dip invertido se ha relacionado con algunas causas secundarias de hipertensión: apnea de sueño, diabetes, preeclampsia, insuficiencia cardíaca, hipotensión ortostática, feocromocitoma, enfermedad de Cushing y trasplante cardíaco.

Algunos autores recomiendan terapia hipotensora en horarios vespertinos $\mathrm{o}$ al acostarse para inducir o profundizar el dip y así revertir su riesgo patogénico ${ }^{18}$.

f) Frecuencia cardiaca

Interesa para elegir la terapia farmacológica. Con alguna frecuencia, cifras muy elevadas están relacionadas con registros erróneos, por lo que deben considerarse cuando existan síntomas o constatación de episodios de taquicardia. La asociación de taquicardia a elevaciones marcadas de presión sugiere secreción episódica de catecolaminas (ej.: feocromocitoma).

Informe y conclusiones: Considerados los elementos anteriores, el informe debe precisar si es:

- Normal: cuando el promedio de las presiones de 24 h y diurnas están dentro de la categoría de normalidad de la Tabla 4.

- Hipertensión arterial: si los promedios antes señalados están anormales según Tabla 4. La clasificación en categorías ha sido controversial, sólo permite encasillar inicialmente a un paciente, pero tiene la ventaja de motivarlo junto al médico tratante para adherir mejor al tratamiento. En una publicación anterior ${ }^{19}$ clasificamos las hipertensiones en 3 categorías. En la experiencia adquirida estos años, estimamos que, según la clasificación del JNC7, se debiera ordenar en etapas 1 y 2 , (anexo 2a)

- La calificación de prehipertensión o borderline sólo permite tomar acciones para iniciar el abordaje del presunto hipertenso y seguramente necesitará repetir la MAPA.

- Las cargas sirven de apoyo al informe y su magnitud debiera correlacionarse con los promedios.
5. Criterios de hipotensión arterial.

La MAPA se realiza también para evaluar hipotensión arterial asociada o no a fármacos. Criterios para considerar hipotensión arterial ${ }^{20}$ :

- Promedio de presiones diurnas: $<110 / 65$ en hombres y $<98 / 61$ en mujeres

- Promedio de presiones nocturnas: $<84 / 45$ (sin diferencias entre sexos).

Otros autores ${ }^{14}$ proponen constatar en al menos 2 registros consecutivos, presiones:

- Diurnas: inferiores a 100/65 mmHg.

- Nocturnas: inferiores a 90/50 mmHg.

6. Re-monitorización de pacientes hipertensos

La monitorización de 24 h debería repetirse a corto plazo cuando hay:

- Alta variabilidad de los registros.

- Respuesta inapropiada al tratamiento.

- Alto riesgo cardiovascular o necesidad de control estricto del tratamiento.

- Cuando persistentemente las presiones de la consulta son altas a pesar de un MAPA normal (controlar la reproducibilidad).

- En caso de hipertensión de "bata blanca" debe repetirse cada 1 ó 2 años o menos si existe alto riesgo cardiovascular (grado de recomendación IIb, evidencia nivel D).

Análisis de la MAPA por el médico tratante junto al paciente

Además del informe del laboratorio que realizó el examen, es de gran utilidad revisar con el paciente la curva de presiones, ya que pueden detectarse situaciones corregibles que gatillan elevaciones tensionales, o alteraciones en el ritmo del sueño que determinen ausencia del dip nocturno.

\section{Grupos especiales}

a) Criterios MAPA en embarazo:

En hipertensas crónicas bajo tratamiento preembarazo es conveniente efectuar MAPA en cada uno de los trimestres del embarazo, para ajustar las dosis de los hipotensores (grado de recomendación IIa).

Los valores de la Sociedad Europea de Hipertensión ${ }^{3}$ y otros alternativos ${ }^{21}$ se expresan en la Tabla 5 .

b) Niños:

En niños la distinción entre hipertensión estable o de "bata blanca" posee una gran relevancia, pues 
Tabla 5. Valores de monitoreo de presión arterial durante los trimestres del embarazo

\begin{tabular}{|c|c|c|c|c|}
\hline \multirow[b]{2}{*}{ PA(mmHg) } & \multicolumn{4}{|c|}{ Gestación (semanas) } \\
\hline & 9-16 & 18-24 & 26-32 & $33-40$ \\
\hline \multicolumn{5}{|l|}{ Diurnas } \\
\hline Sistólica & $115 \pm 8$ & $115 \pm 8$ & $116 \pm 9$ & $119 \pm 9$ \\
\hline Diastólica & $70 \pm 7$ & $69 \pm 6$ & $70 \pm 7$ & $74 \pm 7$ \\
\hline \multicolumn{5}{|l|}{ Nocturnas } \\
\hline Sistólica & $100 \pm 7$ & $99 \pm 8$ & $101 \pm 8$ & $106 \pm 8$ \\
\hline Diastólica & $55+5$ & $54+6$ & $55+6$ & $58+7$ \\
\hline \multicolumn{5}{|c|}{ Puede ser útil además, referirse a promedios de 24 h según lo siguiente ${ }^{13}$ : } \\
\hline Presiones & $1^{\mathrm{er}}$ Trimestre & $\begin{array}{l}\text { Gest } \\
2^{\circ} \text { Trim }\end{array}$ & & $3^{\text {er }}$ trimestre \\
\hline Sistólica & $100,5 \pm 7,7$ & 100,3 & & $104,2 \pm 8,5$ \\
\hline Diastólica & $64,6 \pm 6,1$ & 63,2 & & $65,2 \pm 7,3$ \\
\hline
\end{tabular}

permite evaluar la variabilidad, la eficacia del tratamiento farmacológico y decidir si puede prescindirse de antihipertensivos. Además, para controlar más precisamente las cifras tensionales en patologías pediátricas asociadas a hipertensión (grado de recomendación I, evidencia nivel D).

Debe considerarse para esto, los diferentes puntos de corte de acuerdo a sexo y estatura, estimando hipertensión desde el percentil 95. Se ha propuesto tomar como referencia el percentil 50 como valor promedio normal ${ }^{22}$. En Tabla 6 se ponen estos datos y se han promediado los intervalos de altura ${ }^{23,24}$ (Nivel de evidencia B).

Como no siempre se medirá al paciente, ni se consignará la altura, alternativamente podemos estimar las presiones por edad, Tabla 7 (a $\mathrm{y} b)$.

c) Situaciones especiales relativas a nefropatías, trasplante renal y donantes potenciales:

1) Individuos con deterioro de función renal. Es importante considerar que las patologías renales tienen que estimarse como potencialmente progresivas cuando la filtración calculada por la fórmula abreviada de MDRD es $<60 \mathrm{ml} / \mathrm{min} / 1,73 \mathrm{~m}^{2}$ superficie, o cuando la filtración glomerular es $<90$ $\mathrm{ml} / \mathrm{min} / 1,73 \mathrm{~m}^{2}$ ) en presencia de albuminuria $>30 \mathrm{mg} / \mathrm{g}$ creatinina. Se ha demostrado que el control estricto de las cifras tensionales $(<130 / 80 \mathrm{mmHg})$ es la medida más efectiva para retardar la progresión del daño renal, siendo mejor la asociación de la velocidad de progresión del deterioro de la función renal con presiones ambulatorias que con aquéllas obtenidas en consulta (evidencia nivel $\mathrm{B})^{25}$.

Además, la ausencia del descenso nocturno se asocia a una mayor progresión del deterioro de función renal.

En pacientes con nefropatía diabética, un control estricto de la presión se ve potenciado al bloquear el sistema renina-angiotensina (grado de recomendación I, evidencia nivel A ${ }^{26}$. También, la progresión de su proteinuria se asocia al patrón non-dipper (evidencia nivel B) ${ }^{27}$.

2) Pacientes en diálisis crónica, hipertensos de difícil manejo.

En ellos se recomienda monitorizar durante todo el período interdiálisis. La variación observada del período contribuye a caracterizar la importancia del volumen extrace- 
Tabla 6. Percentiles 50 y 95 (P,50 y P,95) para el promedio de presiones sistólicas y diastólicas de día y noche para niños, estratificadas según sexo y estatura

\begin{tabular}{|c|c|c|c|c|}
\hline \multirow[b]{3}{*}{ Altura (cm) } & \multicolumn{4}{|c|}{$\begin{array}{c}\text { Presiones sistólicas / Presiones diastólicas } \\
\text { Varones }\end{array}$} \\
\hline & \multicolumn{2}{|c|}{ Día } & \multicolumn{2}{|c|}{ Noche } \\
\hline & P. 50 & P. 95 & P. 50 & P. 95 \\
\hline 120 & $112,0 / 73,1$ & $123,5 / 81,2$ & $95 / 55$ & $106,4 / 64,1$ \\
\hline 125 & $112,6 / 73,3$ & $124,0 / 81,3$ & $95,5 / 55,2$ & $107,8 / 64,3$ \\
\hline 130 & $113,0 / 73,4$ & $124,6 / 81,4$ & $96,3 / 55,4$ & $109,5 / 64,5$ \\
\hline 135 & $113,7 / 73,5$ & $125,2 / 81,3$ & $96,7 / 55,5$ & $111,3 / 64,8$ \\
\hline 140 & $114,0 / 73,6$ & $126,0 / 81,2$ & $97,3 / 55,6$ & $113,1 / 65,0$ \\
\hline 145 & $114,5 / 73,7$ & $127,0 / 81,1$ & $98,2 / 55,7$ & $114,7 / 65,2$ \\
\hline 150 & $115,4 / 73,8$ & $128,5 / 81,0$ & $99,0 / 56,0$ & $115,9 / 65,4$ \\
\hline 155 & $116,2 / 73,9$ & $130,2 / 81,1$ & $100,3 / 56,3$ & $117,0 / 65,6$ \\
\hline 160 & $118,0 / 73,9$ & $132,3 / 81,3$ & $102,2 / 56,3$ & $118,0 / 65,7$ \\
\hline 165 & $120,0 / 74,0$ & $134,5 / 81,7$ & $103,4 / 56,7$ & $119,1 / 65,8$ \\
\hline 170 & $121,5 / 74,0$ & $136,7 / 82,2$ & $104,0 / 56,8$ & $120,2 / 65,9$ \\
\hline \multirow[b]{3}{*}{ Altura (cm) } & \multicolumn{4}{|c|}{ Mujeres } \\
\hline & \multicolumn{2}{|c|}{ Día } & \multicolumn{2}{|c|}{ Noche } \\
\hline & P. 50 & P. 95 & P.50 & P. 95 \\
\hline 120 & $111,0 / 72,0$ & $121,1 / 79,7$ & $96,0 / 55,0$ & $109,0 / 66,4$ \\
\hline 125 & $111,6 / 72,4$ & $122,1 / 81,8$ & $96,4 / 55,0$ & $109,8 / 66,2$ \\
\hline 130 & $112,0 / 72,5$ & $123,1 / 81,8$ & $97,0 / 55,0$ & $110,6 / 66,0$ \\
\hline 135 & $113,3 / 72,6$ & $124,1 / 81,8$ & $97,5 / 55,2$ & $111,3 / 65,8$ \\
\hline 140 & $114,0 / 72,6$ & $125,1 / 81,8$ & $98: 2 / 55,3$ & $111,9 / 65,7$ \\
\hline 145 & $114,5 / 72,8$ & $126,3 / 81,8$ & $98,4 / 55,4$ & $112,5 / 65,6$ \\
\hline 150 & $115,0 / 73,0$ & $127,5 / 81,9$ & $99,0 / 55,5$ & $113,1 / 65,5$ \\
\hline 155 & $115,5 / 73,2$ & $128,5 / 81,9$ & $99,5 / 55,5$ & $113,8 / 65,5$ \\
\hline 160 & $116,0 / 73,5$ & $129,3 / 81,9$ & $100,0 / 55,6$ & $114,0 / 65,4$ \\
\hline 165 & $117,0 / 73,6$ & $129,8 / 81,9$ & $100,5 / 55,6$ & $114,0 / 65,2$ \\
\hline 170 & $118,0 / 74,0$ & $130,0 / 81,8$ & $101,0 / 55,7$ & $114,0 / 65,0$ \\
\hline
\end{tabular}

lular y a manejar las dosis de hipotensores para prevenir hipotensión en diálisis con la consiguiente infusión de volumen.

3) Potenciales donantes de riñón.

La presencia de hipertensión agrega un factor de riesgo de deterioro de función renal en monorrenos. Así, reviste especial importancia descartar que un potencial donante sea normotenso en la consulta y tenga una hipertensión enmascarada (evidencia nivel B), pues en este grupo se ha encontrado similar daño orgánico y complicaciones cardiovasculares que para los hipertensos estables. Idealmente deben excluirse como donantes los individuos con hipertensión de "delantal blanco" 28 .

4) Trasplantados renales.

Acarrean factores hipertensógenos por promover deterioro de la función renal, a lo que se agrega el efecto hipertensor de la ciclosporina. 
Tabla 7a. Valores para monitoreo ambulatorio de presión arterial $(\mathrm{mm} \mathrm{Hg})$ para niños por edad (percentiles 50 y 95)

\begin{tabular}{|c|c|c|c|c|c|c|c|c|c|c|c|c|c|}
\hline \multirow{2}{*}{\multicolumn{2}{|c|}{ Percentil }} & \multicolumn{12}{|c|}{ Edad, años } \\
\hline & & 5 & 6 & 7 & 8 & 9 & 10 & 11 & 12 & 13 & 14 & 15 & 16 \\
\hline \multicolumn{14}{|c|}{ PAS/PAD 24-hr } \\
\hline \multirow{2}{*}{\multicolumn{2}{|c|}{ P. 50}} & 104,6 & 105,5 & 106,3 & 107,0 & 107,7 & 108,8 & 110,4 & 112,6 & 115,1 & 117,8 & 120,6 & 123,4 \\
\hline & & 65,3 & 65,7 & 66,1 & 66,3 & 66,5 & 66,6 & 66,9 & 67,2 & 67,4 & 67,7 & 68,1 & 68,6 \\
\hline \multirow{2}{*}{\multicolumn{2}{|c|}{ P. 95}} & 116,4 & 117,7 & 118,9 & 120,0 & 121,1 & 122,5 & 124,6 & 127,1 & 129,9 & 132,7 & 135,5 & 138,2 \\
\hline & & 74,4 & 74,8 & 75,1 & 75,2 & 75,3 & 75,4 & 75,7 & 75,9 & 76,2 & 76,6 & 77,0 & 77,5 \\
\hline \multirow[t]{4}{*}{ Día } & P. 50 & 111,1 & 111,5 & 111,9 & 112,2 & 112,6 & 113,4 & 114,9 & 117,0 & 119,5 & 122,3 & 125,3 & 128,2 \\
\hline & & 72,2 & 72,4 & 72,5 & 72,5 & 72,3 & 72,1 & 72,0 & 72,0 & 72,2 & 72,5 & 73,0 & 73,5 \\
\hline & P. 95 & 122,9 & 123,8 & 124,6 & 125,3 & 126,1 & 127,3 & 129,3 & 131,8 & 134,7 & 137,7 & 140,8 & 143,9 \\
\hline & & 79,1 & 79,3 & 79,7 & 79,8 & 79,7 & 79,5 & 79,5 & 79,5 & 79,7 & 80,0 & 80,6 & 81,3 \\
\hline \multirow[t]{4}{*}{ Noche } & P. 50 & 95,0 & 95,5 & 96,1 & 96,7 & 97,3 & 98,1 & 99,4 & 101,2 & 103,4 & 105,8 & 108,3 & 110,9 \\
\hline & & 55,0 & 55,3 & 55,5 & 55,7 & 55,8 & 55,8 & 55,9 & 56,0 & 56,3 & 56,5 & 56,8 & 57,1 \\
\hline & P. 95 & 106,3 & 108,0 & 109,6 & 111,0 & 112,1 & 113,2 & 114,6 & 116,3 & 118,6 & 121,0 & 123,4 & 125,9 \\
\hline & & 65,1 & 66,1 & 66,8 & 67,1 & 67,1 & 66,9 & 66,7 & 66,5 & 66,5 & 66,5 & 66,4 & 66,4 \\
\hline
\end{tabular}

Tabla $7 \mathrm{~b}$. Valores para monitoreo ambulatorio de presión arterial $(\mathrm{mm} \mathrm{Hg})$ para niñas por edad (percentiles 50 y 95 )

\begin{tabular}{|c|c|c|c|c|c|c|c|c|c|c|c|c|c|}
\hline \multirow{2}{*}{\multicolumn{2}{|c|}{ PA Percentil }} & \multicolumn{12}{|c|}{ Edad, años } \\
\hline & & 5 & 6 & 7 & 8 & 9 & 10 & 11 & 12 & 13 & 14 & 15 & 16 \\
\hline \multicolumn{14}{|c|}{ PAS/PAD 24-hr } \\
\hline \multirow{2}{*}{\multicolumn{2}{|c|}{ P. 50}} & 102,8 & 104,1 & 105,3 & 106,5 & 107,6 & 108,7 & 109,7 & 110,7 & 111,8 & 112,8 & 113,8 & 114,8 \\
\hline & & 65,5 & 65,6 & 65,8 & 65,9 & 66,0 & 66,2 & 66,4 & 66,6 & 67,0 & 67,2 & 67,5 & 67,7 \\
\hline \multirow{2}{*}{\multicolumn{2}{|c|}{ P. 95}} & 114,9 & 116,4 & 117,7 & 118,9 & 120,0 & 121,1 & 122,1 & 123,0 & 123,9 & 124,5 & 125,0 & 125,6 \\
\hline & & 74,0 & 74,1 & 74,2 & 74,2 & 74,4 & 74,7 & 75,1 & 75,6 & 76,1 & 76,4 & 76,6 & 76,7 \\
\hline \multirow[t]{4}{*}{ Día } & P. 50 & 108,4 & 109,5 & 110,6 & 111,5 & 112,4 & 113,3 & 114,2 & 115,3 & 116,4 & 117,5 & 118,6 & 119,6 \\
\hline & & 72,6 & 72,6 & 72,4 & 72,2 & 72,0 & 71,8 & 71,8 & 72,1 & 72,4 & 72,8 & 73,2 & 73,5 \\
\hline & P. 95 & 120,9 & 122,2 & 123,3 & 124,3 & 125,2 & 126,2 & 127,2 & 128,2 & 129,2 & 129,9 & 130,4 & 130,9 \\
\hline & & 82,3 & 82,2 & 82,1 & 81,8 & 81,5 & 81,3 & 81,4 & 81,6 & 82,0 & 82,2 & 82,2 & 82,1 \\
\hline \multirow[t]{4}{*}{ Noche } & P. 50 & 94,8 & 95,6 & 96,2 & 96,8 & 97,5 & 98,2 & 99,0 & 99,7 & 100,5 & 101,3 & 102,0 & 102,9 \\
\hline & & 56,4 & 55,9 & 55,5 & 55,1 & 54,8 & 54,6 & 54,3 & 54,2 & 54,3 & 54,5 & 54,9 & 55,3 \\
\hline & P. 95 & 108,4 & 109,6 & 110,6 & 111,5 & 112,3 & 113,0 & 113,5 & 113,6 & 113,7 & 113,6 & 113,5 & 113,5 \\
\hline & & 68,5 & 67,9 & 67,4 & 66,9 & 66,6 & 66,5 & 66,2 & 65,9 & 65,6 & 65,4 & 65,3 & 65,2 \\
\hline
\end{tabular}

REFERENCIAS

1. Armario P, Hernández del Rey R. Variability of arterial pressure. Nefrologia 2002; 22 Suppl 3: 54-8.

2. Pickering TG, James GD, Boddie C, Harshield GA, Blank S, Laragh JH. How common is white coat hypertension? JAMA 1988; 259: 225-8.
3. Verberk WJ, Kessels AG, de Leeuw PW. Prevalence, causes, and consequences of masked hypertension: a meta-analysis. Am J Hypertens 2008; 21: 969-75.

4. Verdecchia P. Prognostic value of ambulatory blood pressure. Current evidence and clinical implications. Hypertens 2000; 35: 844-51.

5. Omboni S, Parati G, Palatini P, Vanasia A, Muiesan ML, 
Cuspidi C, Mancia G. Reproducibility and clinical value of nocturnal hypotension: prospective evidence from the SAMPLE study. Study on Ambulatory Monitoring of Pressure and Lisinopril Evaluation. J Hypertens 1998; 16: 733-8

6. Еццот WJ. Cyclic and circadian variations in cardiovasular events. Am J Hypertens 2001; $14(9$ Pt 2): 291S-295S.

7. Seo HS, Kang TS, Park S, Choi EY, Ko YG, Choi D et al. Non-dippers are associated with adverse cardiac remodeling and dysfunction (R1). Int J Cardiol 2006; 112: 171-7.

8. CONSENSUS DOCUMENT ON NON INVASIVE AMBULATORY BLOOD PRESSURE MONITORING. The scientific committee. J Hypertens 1990; 8 (suppl 6): s 135-s 140.

9. O’Brien, Asmar R, Beilin L, Imai Y, Mallion JM, Mancia G ET AL. European Society of Hypertension recommendations for conventional, ambulatory and home blood pressure measurements. J Hypertens 2003; 21 : 821-48.

10. Agarwal R, Bunaye Z, Bekele DM. Prognostic significance of between-arm blood pressure differences. Hypertension 2008; 51: 657-62.

11. Winnicki M, Canali C, Mormino P, Palatini P. Ambulatory blood pressure monitoring editing criteria: is standardization needed? Hypertension and Ambulatory Recording Venetia Study (HARVEST) Group, Italy. Am J Hypertens 1997; 10: 419-27.

12. Pickering tG, Hall Je, Appel lJ, Falkner Be, Graves J, Hill MN ET AL. Recommendations for blood pressure measurement in humans and experimental animals: part 1: blood pressure measurement in humans: a statement for professionals from the Subcommittee of Professional and Public Education of the American Heart Association Council on High Blood Pressure Research. Circulation 2005; 111: 697-716.

13. Panagiotakos DB, Kromhout D, Menotti A, Chrysohoou C, Dontas A, Pitsavos C ET al. The relation between pulse pressure and cardiovascular mortality in 12.763 middle-aged men from various parts of the world: a 25-year follow-up of the seven countries study. Arch Intern Med 2005; 165: 2142-7.

14. Hermida RC, Ayala DE, Mojón A, Fernández JR, Silva I, UCIEDA R ET AL. Blood pressure excess for the early identification of gestational hypertension and preeclampsia. Hypertension 1998; 31: 83-9.

15. Tsioufis C, Stefanadis C, Goumas G, Pitsavos C, Toutouzas P. Relation of ambulatory blood pressure load with left ventricular geometry in untreated patients with mild-to-moderate hypertension. J Hum Hypertens 1999; 13: 677-82.
16. Alessi A, Brandão AA, Pierin A, Feitosa AM, Machado CA, De Moraes Forjaz CL et al. IV Guideline for Ambulatory Blood Pressure Monitoring. II Guideline for Home Blood Pressure Monitoring. Arq Bras Cardiol 2005; 85; (Suppl 2): 1-18.

17. Palatini P. Non-dipping in hypertension: still a challenging problem. J Hypertens 2004; 22: 2269-72.

18. Hermida RC, Ayala de, Mojón A, Fernández JR. Chronotherapy with nifedipine GITS in hypertensive patients: improved efficacy and safety with bedtime dosing. Am J Hypertens 2008; 21: 948-54.

19. Prat H, Valdés G, Román O, Zárate lH, Jalil J. Recomendaciones sobre el uso de la monitorización ambulatoria de presión arterial. Documento de consenso de la Sociedad Chilena de Hipertensión. Rev Méd Chile 1999; 127: 1269-73.

20. Owens P, O'Brien E. Hypotension in patients with coronary disease: can profound hypotensive events cause myocardial ischaemic events? Heart 1999; 82: 477-81.

21. Stabouli S, Kotsis V, Zakopoulos N. Ambulatory blood pressure monitoring and target organ damage in pediatrics. J Hypertens 2007; 25: 1979-86.

22. Wuhl E, Witte K, Soergel M, Mehls O, Schaefer F. Distribution of 24-h ambulatory blood pressure in children: normalized reference values and role of body dimensions. J Hypertens 2002; 20: 1995-2007.

23. SAien C. Monitoreo ambulatorio de presión arterial en niños. En: Hipertensión Arterial, cap, 14, pp. 1839. Eds. Saieh C y Zehnder B. Ed. Mediterráneo Ltda, Santiago, Chile, 2008.

24. Urbina E, Alpert B, Flynn J, Hayman L, Harshfield G, JACOBSON M ET AL. Ambulatory Blood Pressure Monitoring in Children and Adolescents: Recommendations for Standard Assessment. Hypertension 2008; 52: 433-51.

25. Agarwal R, Andersen MJ. Prognostic importance of ambulatory blood pressure recordings in patients with chronic kidney disease. Kidney International 2006; 69: 1175-80.

26. Ritz E. Diabetic nephropathy. Saudi J, Kidney Dis Transplant 2006; 17: 481-90.

27. Palmas W, Pickering T, Teresi J, Schwartz Je, Eguchi K, Field L ET AL. Nocturnal blood pressure elevation predicts progression of albuminuria in elderly people with type 2 diabetes. J Clin Hypertens (Greenwich) 2008; 10: 12-20.

28. Ommen ES, Schroppel B, Kim JY, Gaspard G, Akalin E, DE Boccardo G ET al. Routine use of ambulatory blood pressure monitoring in potential living kidney donors. Clin J Am Soc Nephrol 2007; 2: 1030-6. 